\title{
Revealing the Nature and Location of High Energy Emission in the Candidate Binary SMBH System OJ 287
}

\section{Pankaj Kushwaha*, E. M. de Gouveia Dal Pino}

Department of Astronomy (IAG-USP), University of Sao Paulo, Sao Paulo 05508-090, Brazil

E-mail: pankaj.kushwaha@iag.usp.br

\section{Alok C. Gupta}

Aryabhatta Research Institute of Observational Sciences (ARIES),Manora Peak, Nainital 263002, India

\section{Paul J. Wiita}

Department of Physics, The College of New Jersey, P.O. Box 7718, Ewing, NJ 08628-0718, USA

The latest flare of the regular $\sim 12$ years quasi-periodic optical outbursts in the binary SMBH candidate system OJ 287 occurred in December 2015. Following this, the source has exhibited enhanced multi-wavelength (MW) variability in spectral, temporal and polarization domains with new features never seen before. Our MW investigation show that the overall MW variability can be divided into two-phase, (i) November 2015 - May 2016 with variability from near-infrared (NIR) to Fermi-LAT $\gamma$-ray energies $(0.1-300 \mathrm{GeV})$, and (ii) September 2016 - July 2017 with intense NIR to X-ray variability but without any activity in the Fermi-LAT band, and the very first detection at very high energies (VHEs, E > $100 \mathrm{GeV}$ ) by VERITAS. The broadband SEDs during the first phase show a thermal bump in the NIR-optical region and a hardening in the $\gamma$-ray spectra with a shift in its peak. The thermal bump like feature is consistent with the description of the standard accretion-disk associated with the primary SMBH of mass $\sim 1.8 \times 10^{10} M_{\odot}$ while the $\gamma$-ray emission can be naturally reproduced by inverse Compton scattering of photons from the broad line region which has been seen during the close encounter duration of the binary SMBHs, thereby suggesting a sub-parsec scale origin. The SEDs during the second phase (VHE detection) is a mixture of typical OJ 287 SED with hardened $\gamma$-ray spectra and an HBL SED and can be explained in a two-zone model, one located at sub-parsec scales and other at parsec scales. During both the phases, the MW variability is simultaneous and almost always accompanied by changes in the polarization properties, exhibiting random and systematic variations, suggesting a strong role of magnetic field and turbulence.

International Conference on Black Holes as Cosmic Batteries: UHECRs and Multimessenger Astronomy BHCB2018

12-15 September, 2018

Foz du Iguazu, Brasil

\footnotetext{
* Speaker.
} 


\section{Introduction}

OJ 287 belongs to the BL Lac (BLL) subclass of blazars - active galactic nuclei with relativistic jet directed at close angles to our line of sight and characterized by featureless emission spectra or a weak signal of optical emission lines superimposed on it. Identified in 1967 [1] and located at the redshift of $\mathrm{z}=0.306[2,3]$, its radio and optical brightness combined with a diverse range of peculiar properties have made it one of the best-studied sources across the entire electromagnetic (EM) spectrum $[4,5$, and references therein $]$ and a potential target for many of the unresolved problems in relativistic jet physics $[6,5,7]$ as well as general relativity [8, and references therein]. The source central engine has been argued to be a precessing binary supermassive black hole (SMBH) system of masses $1.8 \times 10^{10} M_{\odot}$ and $1.5 \times 10^{8} M_{\odot}$ from an $\sim 11.65$-year periodicity in the optical light curve [8, and references therein]. Though the model has undergone many iterations from its first inception, the basic tenets and claim remain the same. It predicted the next outburst between the end of 2015 and the beginning of 2016 depending on the spin of the primary SMBH. Contrary to this, radio kinematic studies suggest $\sim 22$-year periodicity and rather argue and favor a jet-disk central engine with jet rotating around its axis and precessing as well [6, and references therein].

One of the fundamental problems in the blazars' research is the process/mechanism responsible for the high-energy hump in their characteristic broad double-humped spectral energy distributions (SEDs). Broadly two possible channels: leptons and hadrons are the primary candidates. In the former, the high-energy hump originates as a result of inverse Compton (IC) scattering of the local soft photons fields while it is due to proton-proton and/or proton-photons initiated cascade processes in the case of the latter. The widely considered target photon fields for the leptonic channel includes the synchrotron photons within the jet and accretion disk (AD), broad line region (BLR), IR torus (IR), and cosmic microwave background photons (CMB) external to the jet. The IC scattering of synchrotron photons is called synchrotron self-Compton (SSC) while IC of an external field is generally called external Comptonization (EC) with EC-AD, EC-BLR, EC-IR, and EC-CMB representing the respective IC spectrum of the mentioned external fields. The lowenergy component of the SED which spans radio to ultraviolet (UV) and even to X-ray energies is widely regarded as synchrotron emission from relativistic non-thermal electrons in the jet due to its high and variable polarization. Temporally, flux variability is stochastic, present on all time-scales from minutes to decades with statistical properties broadly consistent with other accretion-powered sources [9, 10], and often accompanied by changes in spectral and polarization properties.

The presence of synchrotron emitting relativistic electrons makes IC process as one of the natural candidates for the origin of the high-energy component of the blazars' SEDs. In this scenario, the high-energy component of BLLs is widely attributed to the SSC process due to the lack of strong photon fields external to the jet. The BLL object OJ 287, from the perspective of broadband SED, belongs to the low-frequency peaked BLL with the low-energy component peak at or below NIR bands and the high-energy peak at $100 \mathrm{MeV}$ or below [11, 7]. Detailed modeling of source's broadband SEDs by [7] during one of the best observed multi-wavelength (MW) flares in 2009 showed that observational constraints from the different observed flux states rules out the SSC process for MeV-GeV $\gamma$-ray origin. Rather it requires both SSC (for X-ray) and EC-IR from a torus of temperature of $\sim 250 \mathrm{~K}$ to explain the second energy component [7].

In this proceeding, we report the results of MW spectral and temporal analysis of the two of 
the outburst phases shown by OJ 287 between end 2015 to mid-2017 as reported in $[4,5]$.

\section{Multi-wavelength data}

Since the outburst was anticipated as per the binary SMBH model, OJ 287 was under monitoring and was followed by a large number of observatories in a coordinated fashion as soon as the first sign of an increase in activity was observed [8, 12]. A typical trend of multi-band flux variability observed following the first sign of increased activity till mid-2017 (15 October $2015-26$ June 2017) is shown in figure 1 by the $\gamma$-ray and X-ray light curve from Fermi-LAT and Swift-XRT respectively. The flux variability trend in NIR to ultraviolet (UV) bands was similar to the X-rays. The full MW light curves and the details of observations are presented in the [12, 4, 5, 13].

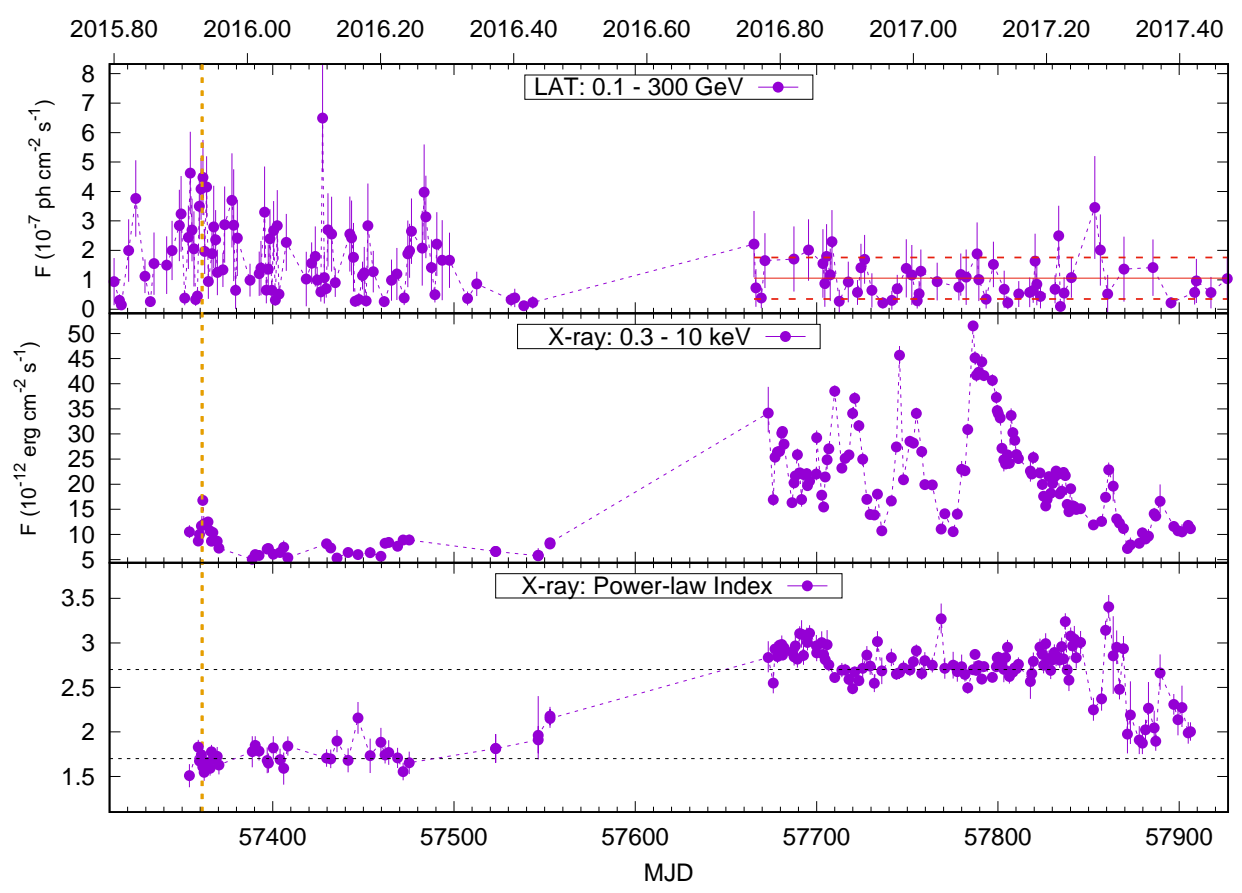

Figure 1: The $\gamma$-ray and X-ray light curves of OJ 287 between 15 October 2015 to 26 June 2017 along with the corresponding X-ray power-law index. The $\gamma$-ray points are extracted from Fermi-LAT data binned over 1-day while the X-ray data correspond to the different observation IDs of Swift-XRT. The NIR-optical flux variability trend is similar to the X-ray $[4,5$, for more details]. The vertical line marks the impact outburst [8] and solid red horizontal line in the top panel represent the flux mean over the plotted duration while the dotted lines show the mean flux-error around it.

\section{Discussion}

The rise of NIR-optical emission arrived around the time predicted by the binary SMBH model and thus was followed across the EM spectrum in a coordinated way by different groups and observatories [8, 13]. Since then till mid-2017, OJ 287 has shown two phases of high MW activity, both characteristically different from each other as well as with all the previously reported short duration MW activity in terms of flux variability and the polarization properties. The first phase 
corresponds to the activity seen during and after the impact outburst till the source flux almost attained the pre-outburst level, roughly November 2015 - May 2016 (MJD 57315 - 57460) period $[13,5]$. The outburst at MJD 57361, marked by the vertical line in figure 1 is the claimed impact outburst resulting from the impact of secondary SMBH on the accretion disk of the primary [8]. It has a relatively low degree of polarization $(<10 \%)$ but marks the beginning of a huge systematic swing of polarization angle by $\sim 200^{\circ}$ [13]. It continued to be observed in the optical even after it reached its pre-outburst flux level, but only in a few bands [13], until the yearly gap when it becomes too close to the Sun. As soon as observations were resumed following the yearly gap, it was already in a high NIR-optical flux states and thus, was observed across the EM bands $[12,4]$. Observations in X-ray showed it to be in a historic high state and thus was observed by the VERITAS under the target of Opportunity source which resulted in its first-ever detection at VHEs [14].

In terms of spectral and temporal MW trends so far reported in the literature over a short duration, the current two phases are unique in many ways. Temporally, the MW flux variations seen during the first phase are similar to previously reported MW activity during the flares with flux variations from NIR to $\mathrm{MeV}-\mathrm{GeV} \gamma$-rays and all being simultaneous within their respective observational cadence $[5,7]$. The variations are associated with frequent and strong changes in optical PA and PD. On the contrary, the MW flux variability during the second phase displays an unreported combination of observations where OJ 287 showed intense NIR to X-ray variation but statistically no variability in $\mathrm{MeV}-\mathrm{GeV}$ energy band though it is detected quite frequently over daily binning of the data. During this phase too, the NIR to X-ray variations are simultaneous for all except towards the end duration, during which it attained its lowest flux level, similar to the low-level flux of the first phase. This period instead suggests a hint of systematic variation with flux rise appearing first at high energies and then subsequently at lower energies. The polarization variations are also quite different with PD variation being like that in the first phase, while the PA changed rather smoothly and systematically by $\sim 100^{\circ}$ with fluctuations superposed on it over the duration of the second phase [4].

From energy spectral point of view too, the two duration are completely different with respect to each other (see figure 2) as well as with all the previously reported broadband SEDs of the source in literature till date [7,11]. For the first phase, the broadband SED constructed for the impact flare which has a good coverage from NIR to $\mathrm{MeV}-\mathrm{GeV} \gamma$-rays shows a break in the NIRoptical spectrum and also a hardening of the $\mathrm{MeV}-\mathrm{GeV}$ spectrum with a shift in its peak to higher energies [5]. The MeV-GeV spectral hardening can also be seen in the broadband SED constructed for the low flux state which, unfortunately, lacks the NIR-optical data. Either of the broadband SED, however, show no change in the X-ray spectrum. Focusing on the spectral shape of NIR data ( $\mathrm{J}$ and $\mathrm{K}$ bands) of impact SED clearly show that there is no shift in the location of NIR-optical peak with respect to the 2009 NIR spectra and thus suggest that the NIR-optical break is likely an additional emission component. On the other hand, in the leptonic emission scenario, the spectral shape and the location of SED peaks of the low-energy component is related to the corresponding spectral shape and the location of peak of the high-energy component. Thus, the lack of change in the NIR-optical spectrum and its peak location rules out the previous EC-IR of a $\sim 250 \mathrm{~K}$ torus interpretation [7] for the current $\mathrm{MeV}-\mathrm{GeV}$ spectral hardening and peak shift. Combining the no shift and change in the NIR part with no change in X-rays suggests the $\mathrm{MeV}-\mathrm{GeV}$ emission to arises from a different process or IC of photon field. Surprisingly, line emission in OJ 287 has been 


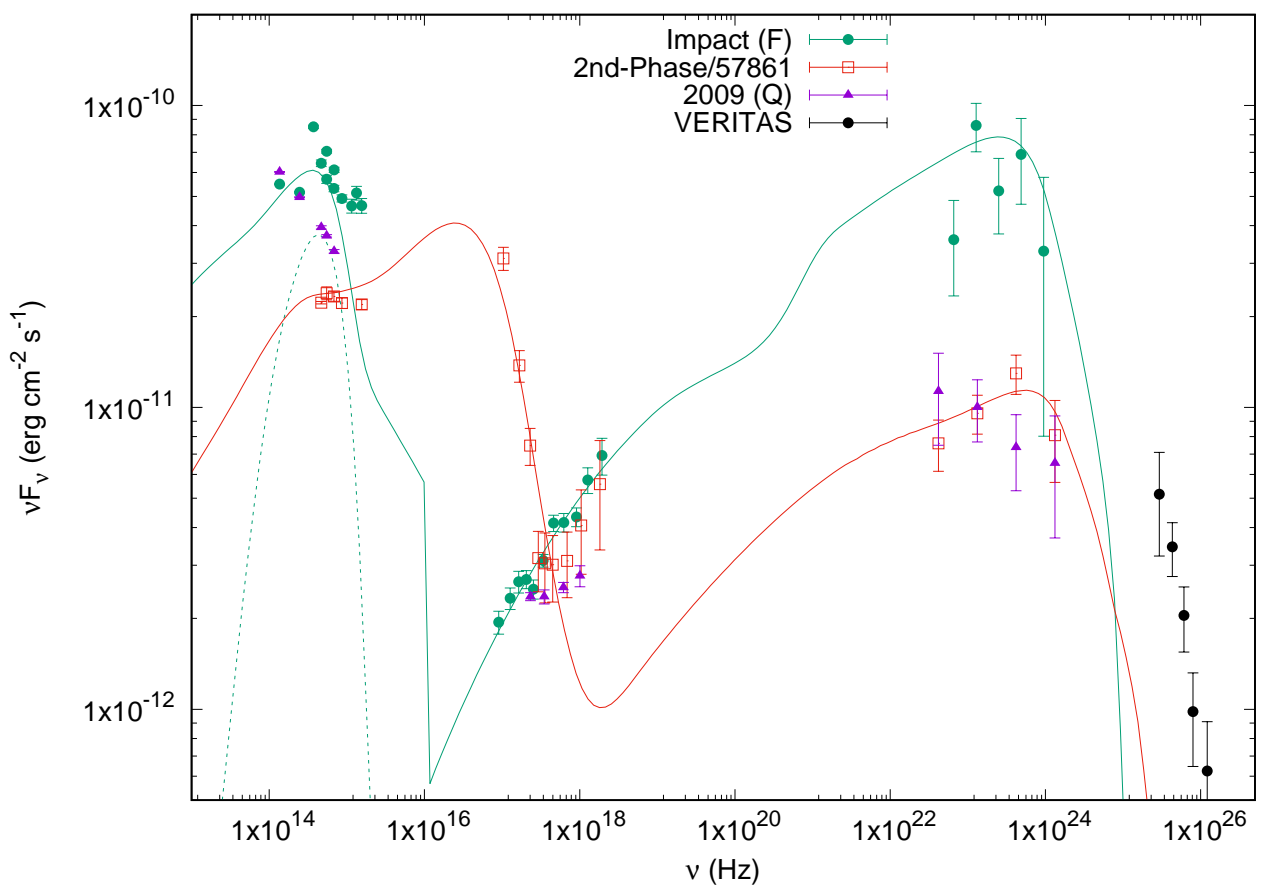

Figure 2: Broadband SEDs of OJ 287 corresponding to different flux states (Flare: F, quiescent: Q) of the source.

detected during the impact duration of 1984 and 2005 - 2008 [15] and the first phase is one such. Addition of a BLR-IC component naturally reproduces both the $\mathrm{MeV}-\mathrm{GeV}$ spectral hardening as well as the shift in its peak while at the same time is consistent with the NIR shape and its peak location. This, in addition, indirectly constrains the location of the emission region to be within BLR region which is on sub-parsec scales, contrary to the previous claim of parsec-scale location [7]. On the other hand, the NIR-optical break is naturally produced by the accretion-disk emission associated with the primary SMBH of mass $\sim 1.8 \times 10^{10} M_{\odot}$ [5]. Surprisingly, its first appearance in end May 2013 (MJD 56439) is temporally coincident with the time of impact of the secondary in the SMBH frame as predicted by the binary SMBH model [8], thereby favoring a binary SMBH central engine over other claims/interpretations [6].

As far as the spectral evolution during the second phase is concerned, the broadband SEDs during the high optical to X-ray flux states are superposition of the typical broadband SED of OJ 287 with the modified $\mathrm{MeV}-\mathrm{GeV}$ spectrum and a high-frequency peaked BLL (HBL) SED while the low flux or quiescent state SED for this duration is similar to the low flux SED of the previous phase. Further, the LAT spectrum for the VHE episode is consistent with the extrapolation of the VERITAS VHE spectrum to the LAT band [4]. Focusing on the departure of the high-energy end of X-ray spectrum with respect to its lower-energy end and its level to that of the quiescent $\mathrm{X}$-ray spectrum from the first phase, one can clearly see that this new high $\mathrm{X}$-ray emission is nothing but an additional HBL component with its peak in UV to soft X-ray region (see fig. 2, [4]). Accordingly, the overall SED can be modeled with a two-zone model with the first zone responsible for the typical OJ 287 SED with modified MeV-GeV spectrum while the other zone produces an HBL like SED, resulting from the SSC + EC-IR [4]. 
The non-variability of MeV-GeV band can also be clearly understood from the extracted SEDs during the second phase. Since the photon-flux is based on counting, the overall integrated photon count over the instrument band is dominated by the photons at the low-energy end for sources with power-law emission as typically seen in nature. Focusing over the first data point at MeV-GeV $\gamma$-ray band which is consistent with the corresponding 2009 quiescent state flux (see fig. 2) while the peak SED point is at most $\sim 1.5$ times to its 2009 value. This translates at most to a similar increment of photons in the energy band of the peak point (energy deposited $\propto$ number of photons). Thus, the order of magnitude difference in the energy of the two bands transforms to a 4 order of magnitude difference in the respective photon counts assuming a power-law spectral index of 2 and hence, no appreciable change in the observed photon flux vis-a-vis 2009 quiescent photon flux.

To sum up, the MW activity seen during 15 October 2015 - 26 June 2017 revealed a completely new spectral and temporal phase of OJ 287 and resolved the fundamental problem of nature of $\mathrm{MeV}-\mathrm{GeV}$ emission in OJ 287 and indirectly provided the location of emission region. The association of most of the flux changes with PD and a systematic as well as fluctuating change in PA suggests a strong role of the magnetic field and turbulence for the observed features. The activity and its occurrence close to the timing predicted by the binary SMBH model makes OJ 287 as one of the best potential candidates to explore SMBH Jet-disk connection and tie down high energy emission processes within the central engine.

\section{Acknowledgments}

PK and EMDGP acknowledge support from the Brazilian agencies FAPESP (grant 2015/139330 and 2013/10559-5) and CNPq (grant 308643/2017-8).

\section{References}

[1] J. R. Dickel, K. S. Yang, G. C. McVittie and G. W. Swenson, Jr., A survey of the sky at 610.5 MHz. II. The region between declinations +15 and +22 degrees., AJ 72 (1967) 757 .

[2] M. L. Sitko and V. T. Junkkarinen, Continuum and line fluxes of OJ287 at minimum light, PASP 97 (1985) 1158.

[3] M. Stickel, J. W. Fried and H. Kuehr, Optical spectroscopy of 1 Jy BL Lacertae objects and flat spectrum radio sources, A\&AS 80 (1989) 103.

[4] P. Kushwaha, A. C. Gupta, P. J. Wiita, M. Pal, H. Gaur, E. M. de Gouveia Dal Pino et al., The ever-surprising blazar OJ 287: multiwavelength study and appearance of a new component in X-rays, MNRAS 479 (2018) 1672 [1803.10213].

[5] P. Kushwaha, A. C. Gupta, P. J. Wiita, H. Gaur, E. M. de Gouveia Dal Pino, J. Bhagwan et al., Multiwavelength temporal and spectral variability of the blazar OJ 287 during and after the 2015 December flare: a major accretion disc contribution, MNRAS 473 (2018) 1145 [1709. 04957 ].

[6] S. Britzen, C. Fendt, G. Witzel, S. J. Qian, I. N. Pashchenko, O. Kurtanidze et al., OJ287: deciphering the 'Rosetta stone of blazars, MNRAS 478 (2018) 3199.

[7] P. Kushwaha, S. Sahayanathan and K. P. Singh, High energy emission processes in OJ 287 during 2009 flare, MNRAS 433 (2013) 2380 [1305. 5065 ]. 
[8] M. J. Valtonen, S. Zola, S. Ciprini, A. Gopakumar, K. Matsumoto, K. Sadakane et al., Primary Black Hole Spin in OJ 287 as Determined by the General Relativity Centenary Flare, ApJL 819 (2016) L37.

[9] P. Kushwaha, A. Sinha, R. Misra, K. P. Singh and E. M. de Gouveia Dal Pino, Gamma-Ray Flux Distribution and Nonlinear Behavior of Four LAT Bright AGNs, ApJ 849 (2017) 138 [1 709.07904 ].

[10] P. Kushwaha, S. Chandra, R. Misra, S. Sahayanathan, K. P. Singh and K. S. Baliyan, Evidence for Two Lognormal States in Multi-wavelength Flux Variation of FSRQ PKS 1510-089, ApJL 822 (2016) L13 [1604.04335].

[11] A. A. Abdo, M. Ackermann, I. Agudo, M. Ajello, H. D. Aller, M. F. Aller et al., The Spectral Energy Distribution of Fermi Bright Blazars, ApJ 716 (2010) 30 [0912 . 2040 ].

[12] A. C. Gupta, H. Gaur, P. J. Wiita, A. Pandey, P. Kushwaha, S. M. Hu et al., Multiband optical flux and polarization variability of the blazar OJ 287 during 2016 - 2017, ArXiv e-prints (2018) arXiv:1803.03964 [1803.03964].

[13] A. C. Gupta, A. Agarwal, A. Mishra, H. Gaur, P. J. Wiita, M. F. Gu et al., Multiband optical variability of the blazar OJ 287 during its outbursts in 2015-2016, MNRAS 465 (2017) 4423 [1611. 07561 ].

[14] S. O'Brien, VERITAS detection of VHE emission from the optically bright quasar OJ 287, ArXiv e-prints (2017) arXiv:1708.02160 [1708.02160].

[15] K. Nilsson, L. O. Takalo, H. J. Lehto and A. Sillanpää, H-alpha monitoring of OJ 287 in 2005-08, $A \& A 516$ (2010) A60 [1004.2617]. 